Revue québécoise de psychologie

$W_{\text {québécoî̀se }}^{\text {Revue }}$

VÉCU PSYCHIQUE DU TRAUMA DE LA MALTRAITANCE INFANTILE ET RÉSILIENCE SORORALE : LECTURE CLINIQUE ET PROJECTIVE

EXPERIENCING CHILD ABUSE TRAUMA AND RESILIENCY IN A SORORAL GROUP: CLINICAL UNDERSTANDING AND PROJECTIVE PSYCHOLOGY CONTRIBUTION

\author{
Anne-Valérie Mazoyer, Séverine Auriol-Verge, Marjorie Roques et Brune De \\ Berail
}

Volume 37, numéro 1, 2016

URI : https://id.erudit.org/iderudit/1040111ar

DOI : https://doi.org/10.7202/1040111ar

Aller au sommaire du numéro

Éditeur(s)

Revue québécoise de psychologie

ISSN

2560-6530 (numérique)

Découvrir la revue

Citer cet article

Mazoyer, A.-V., Auriol-Verge, S., Roques, M. \& De Berail, B. (2016). VÉCU PSYCHIQUE DU TRAUMA DE LA MALTRAITANCE INFANTILE ET RÉSILIENCE SORORALE : LECTURE CLINIQUE ET PROJECTIVE. Revue québécoise de psychologie, 37(1), 225-250. https://doi.org/10.7202/1040111ar

\section{Résumé de l'article}

Après avoir rappelé les principales théories du traumatisme, nous analysons les effets psychiques des maltraitances et la mobilisation de la résilience de type sororal auprès de 3 jeunes soeurs placées dans un foyer éducatif. Grâce à une méthodologie projective (entretiens cliniques de recherche, Children apperception test et un dessin de la famille), les résultats montrent que le trauma peine à être intégré par la fratrie et que la résilience sororale ne s'est pas développée. Cet article affirme la nécessité de soutenir une réflexion autour de placement de membres d'une même fratrie. 


\title{
VÉCU PSYCHIQUE DU TRAUMA DE LA MALTRAITANCE INFANTILE ET RÉSILIENCE SORORALE : LECTURE CLINIQUE ET PROJECTIVE
}

EXPERIENCING CHILD ABUSE TRAUMA AND RESILIENCY IN A SORORAL GROUP: CLINICAL UNDERSTANDING AND PROJECTIVE PSYCHOLOGY CONTRIBUTION

\author{
Anne-Valérie Mazoyer ${ }^{1}$ \\ Université Jean Jaurès (Toulouse) \\ Séverine Auriol-Verge \\ Marjorie Roques \\ Université de Caen (Caen) \\ Université Jean Jaurès (Toulouse) \\ Brune De Berail \\ Université Jean Jaurès (Toulouse)
}

Avec la clinique contemporaine, le psychologue est confronté à diverses situations traumatogènes qui lui imposent une réflexion sur le cadre de la rencontre clinique et ses visées (diagnostique, prise en charge psychothérapeutique et orientations).

Cet article tente de comprendre les processus traumatiques chez l'enfant et de réfléchir au traitement psychique de cette population. Le trauma à l'œuvre chez l'enfant maltraité fait obstacle au travail de représentation en produisant une sidération et déclenche la mise en place de défenses drastiques comme le déni, le clivage. Selon Berger (1992), les expériences inadaptées et angoissantes ont des répercussions sur les trois grandes lignées constitutives de la construction de la personnalité du sujet, à savoir la représentation de soi, la constitution du narcissisme et le traitement psychique des pulsions sexuelles et agressives. Les études prospectives et rétrospectives sur les effets de la maltraitance mettent en avant des retentissements psychiques, somatiques et sociaux sur le longterme (Benarous, Consoli, Raffin, \& Cohen, 2014). La clinique nous enseigne cependant que malgré des relations empreintes de violences, de ruptures, de carences, et en dépit d'un contexte familial défaillant, l'enfant peut dépasser ce vécu traumatique et développer des capacités d'adaptation s'apparentant alors au processus de résilience. Notre clinique nous amène à nous interroger sur le développement psychique d'enfants exposés à diverses formes de maltraitances (psychiques, physiques, sexuelles) et à repérer leurs ressources psychiques et les traces laissées par le trauma. Notre intérêt porte également sur le rôle que peut jouer la fratrie et les effets du placement sororal (Arnaud, 2003) dans le traitement du traumatisme. En analysant trois études de cas fillettes âgées de 7, 9 et 10 ans, issues d'une même fratrie placée dans le même foyer éducatif suite à des négligences parentales, à des violences conjugales et à des suspicions de violence sexuelle, grâce à une méthodologie de recherche comprenant deux épreuves projectives (dessin de la famille et CAT), nous

1. Adresse de correspondance: Université Jean Jaurès, 5 allées Antonio Machado, 31058 Toulouse cedex 9, France. Courriel : avaleriemazoyer@orange.fr 
réfléchirons au processus d"intégration du trauma et à la mobilisation de la résilience (Cyrulnik, 1999).

\section{PERSPECTIVES HISTORIQUES DU TRAUMA EN PSYCHANALYSE}

Initialement, le trauma et le traumatisme appartiennent au registre du médical. Si le trauma s'entend comme une blessure avec effraction, le traumatisme renvoie aux effets durables sur l'organisation de la personnalité et aux moyens défensifs mis en place pour juguler ce trauma (Laplanche, Pontalis, 1967). Au début de la psychanalyse, le trauma réel apparaît comme un facteur étiologique de la névrose pour laisser place au fantasme (abandon de la neurotica). La première guerre mondiale va amener Freud, s'intéressant à la névrose de guerre, à reconsidérer le rôle du trauma provenant d'une source externe. En 1920, dans Au-delà du principe de plaisir, le traumatisme se définit comme une effraction du pareexcitation, mettant hors-jeu le principe de plaisir au profit d'une contrainte à la répétition (compulsion de répétition). Puis en 1926, Freud conceptualise que le trauma appartient tant au registre du dedans que du dehors, le moi étant tout autant attaqué par l'extérieur que par les pressions pulsionnelles internes. La controverse entre Freud et Ferenczi a comme objet la place donnée au fantasme ou à l'événement réel mais aussi l'importance accordée à la sexualité. L'objectif de Freud était d'unifier les différentes névroses (de guerre, traumatiques, classiques) en rappelant l'importance de la vie sexuelle.

Par contre, la clinique auprès des blessés de guerre de Ferenczi (1919) a mis en évidence le rôle du narcissisme et de la régression : le sujet traumatisé se retire du monde et la sexualité est mise hors-jeu). II interroge à nouveau la réalité du traumatisme (1934) à partir des discours de ses patients dits " victimes. Pour lui, l'événement traumatique précoce et vécu par les sujets, résulterait d'une confusion de langues entre l'enfant et les adultes, d'une séduction incestueuse (1932). Le langage de la tendresse représentant la sexualité infantile rencontre le langage passionnel de l'adulte dont la sexualité empreinte d'érotisme est susceptible de pervertir et de culpabiliser l'enfant. Selon Ferenczi, cette réponse affective inadéquate ou l'absence de réponse de l'objet contribue à maintenir une souffrance psychique liée à l'intériorisation de cet objet primaire défaillant, provoquant de graves blessures narcissiques. Une sensation de détresse primaire peut alors se réactiver à tout moment de vulnérabilité. Ferenczi insiste sur l'idée que c'est surtout le désaveu, autrement dit, la non reconnaissance de la souffrance psychique de l'enfant et la disqualification de l'affect ressenti, qui s'avèrent traumatiques. II évoque le terrorisme de la souffrance ayant pour conséquences une paralysie psychique, une altération de la pensée et de la perception et un démantèlement des contenants psychiques, des limites du dehors et du 
dedans (ou collusion entre fantasme et réalité ce que Janin en 1996 nomme le collapsus interne). L'idée d'une fragmentation du Moi développée par Ferenczi (1934), concerne l'existence d'un clivage pour survivre au traumatisme, le sujet se divise en une partie qui souffre et qui ne le sait pas et une autre partie qui sait mais ne souffre pas, les deux tendant à s'ignorer.

\section{CONCEPTIONS ACTUELLES DU TRAUMATISME}

Taïeb, Baubet, Pradère, Lévy, Revah-Lévy, Serre et Moro (2004) rappellent qu'il existe trois modèles de compréhension du traumatisme : celui de la névrose traumatique développé par la psychanalyse (lequel est notre référentiel de prédilection), le modèle cognitif qui pointe les troubles de la mémoire autobiographique et une perspective neurodéveloppementale qui reconnaît les effets des traumas précoces sur la maturation cérébrale et les systèmes impliqués dans la régulation du stress. Les horreurs perpétrées durant la seconde guerre mondiale et les effets sur les civils puis les atrocités liées à la guerre du Vietnam, ou encore plus récemment les catastrophes collectives et les terrorismes idéologiques ont contribué à édifier des nouvelles théorisations du traumatisme en vue de prévenir ses effets. Terr (1991) distingue le traumatisme de type I, qui renvoie à l'exposition soudaine et unique à un événement traumatique, et le traumatisme de type II qui correspond à une répétition d'événements (chronicité). Des symptômes s'avèrent communs aux deux types de traumas comme les reviviscences (qui ne sont pas des souvenirs), des comportements répétitifs, des peurs et des changements d'attitude envers le monde et, certains symptômes sont spécifiques au II comme des états de dépersonnalisation, une anesthésie affective, l'amnésie... Cependant, cette conceptualisation présente quelques limites si nous considérons que ce n'est pas l'événement à lui seul qui crée le trauma mais la manière dont le sujet va se l'approprier et y répondre.

Après un événement traumatique peut se constituer un état de stress post traumatique (ESPT) qui présente diverses manifestations psychopathologiques: l'évitement pouvant conduire à un émoussement émotionnel, la répétition (cauchemars répétitifs, hallucinations), l'hypervigilance provoquant des troubles du sommeil, de la concentration, de l'hyperactivité. La comorbidité de différents troubles comme les troubles de l'attention, des conduites, ou encore oppositionnels avec l'ESPT est également à souligner. Taïeb et al (2004) soulignent néanmoins que I'ESPT reconnu dans le DSM et dans la CIM est insuffisant à rendre compte de la complexité du trauma en clinique et que d'autres facteurs individuels, familiaux, culturels sont à considérer, comme la nature de l'expérience traumatique, le développement du sujet, la qualité de ses interactions et les réponses de l'environnement. Ils précisent que les 
différentes thérapeutiques et orientations doivent tenir compte de toutes ces dimensions. En outre, l'âge et le niveau de développement semblent jouer un rôle sur le type d'expression symptomatique des troubles et non sur leur prévalence (Bailly, 2001). Certains troubles manifestés par les enfants semblent spécifiques selon les âges : les petits d'âge préscolaire vont présenter des conduites régressives touchant le sommeil ou encore des manifestations somatiques. Les enfants dans la phase de latence développent plus de symptômes anxieux ou dépressifs, tandis que les adolescents ont plus largement recours à des substances psychoactives et à des comportements auto ou hétéro-agressifs. Dans tous les cas, il semblerait que les parents comme le groupe familial et social puissent amortir le choc psychique (American Academy of Child and Adolescent Psychiatry Official Action, 1998).

\section{DÉFINITION DE LA RÉSILIENCE}

Le trauma ne peut être envisagé exclusivement sous l'angle de la psychopathologie, tel que le modèle de la résilience le montre. En effet, la résilience ne s'inscrit pas dans une logique de la vulnérabilité, elle relève de facteurs de protection, elle constitue pour le sujet une manière de développer de nouvelles capacités et compétences. Cyrulnik (1999) postule une adaptation possible, une résilience de l'enfant face à l'adversité. La résilience renverrait à la résistance, à la reconstruction, à la mobilisation de ressources, à la capacité à métaboliser l'événement pour continuer à vivre (Anaut, 2002 ; Manciaux, 1993). Les théories actuelles soulignent que la résilience relève d'une approche plus descriptive que conceptuelle (Lighezzolo, Marchal et Theis, 2003) : cette notion peut tout autant être envisagée comme un phénomène, une forme de développement, que comme une capacité ou un processus. Selon nous, la résilience s'apparente à un processus car elle n'est jamais donnée d'avance. Elle se développe différemment d'un sujet à un autre et l'envisager en termes de processus dynamique et évolutif, nous renvoie à la variabilité interindividuelle qui fait que chacun, dans sa singularité, aura à faire à sa propre histoire, avec ses ressources et ses fragilités. La résilience suppose une confrontation au traumatisme et correspond au fait que le sujet résiste lors de la phase paroxystique, s'en protège et la traite, ce que Lemay (2001, p. 135) décrit comme un «tremplin permettant de rebondir dans la vie $»$.

Dans une approche multifactorielle, interactionnelle et non déterministe, Lighezzolo et al. (2003) déclinent en premier lieu les facteurs de protection qui influencent les réponses des sujets confrontés à des événements douloureux comme la maltraitance. Ils définissent des facteurs de protection interne englobant les caractéristiques de la personnalité résiliente (mentalisation) et le rôle souple ou rigide des 
mécanismes de défense (ceux-ci pouvant activer ou au contraire freiner la résilience). La mentalisation peut être définie comme l'habileté à comprendre, chez soi et chez les autres, les états émotionnels, que sont les intentions, désirs, pensées et affects qui sous-tendent les comportements (Fonagy, Target, 2006). Elle est étroitement liée à la qualité des interactions avec les principales figures d'attachement (Fonagy, Gergely, Jurist et Target, 2004) car pour conférer un sens à la pensée de l'autre, l'enfant a rencontré un adulte capable de s'adapter à son expérience subjective (Fonagy, Steele, Steele, Moran, Higgitt, 1991), de décrypter les émotions et les états affectifs de son enfant au risque que ne se produisent un "trauma relationnel " altérant ses capacités de mentalisation (Scheeringa, Zeanah, 2001).

Après avoir exposé des facteurs de protection interne, penchons-nous dès lors sur les facteurs de protection externe (Lighezzolo et al. 2003) ce que Cyrulnik (1999): nomme «tuteurs de résilience ${ }^{1}$ ». A partir de situations de placement ou d'adoption de fratries, Arnaud (2003) présente le groupe fraternel comme une entité pouvant suppléer les défaillances parentales. Dans les cas de fratries placées en foyer d'accueil, il est fréquent de constater un maintien du lien fraternel permettant d'assurer et d'assumer la continuité du groupe familial. Dans ces situations singulières de séparation ${ }^{2}$, d'isolement, de rupture, l'on observe que les liens fraternels souffrent certes, mais s'unifient. Souvent, en réponse à un vécu traumatique, la fratrie développe une qualité nouvelle d'attachement entre ses membres et crée une enveloppe fraternelle contenante, protectrice, voire restauratrice. De nouveaux liens s'organisent souvent selon la place que leurs parents leur avaient accordée ou refusée. Le groupe fraternel instaure une nouvelle donne familiale, repérable par la fluctuation des places et rôles dans la fratrie, qui pallie la perte des figures parentales ou tente de la nier. II peut permettre de rétablir la continuité de la transmission de l'histoire familiale et favorise ainsi un étayage identitaire, constituant une forme de résilience. Ainsi au cours du placement, est observé combien le groupe fraternel fait corps (union groupale) contre un extérieur perçu et vécu comme menaçant.

1. On s'attend de ce tuteur à ce qu'il fasse montre de qualités de présence, de constance, de disponibilité et d'étayage. Ce lien intersubjectif favorise le développement psychoaffectif de l'enfant.

2. Selon Arnaud (2003), l'investissement narcissique du lien fraternel contient et jugule l'effet traumatique de la séparation. 


\section{MÉTHODOLOGIE}

\section{Réflexions déontologiques et contexte de la recherche}

Au niveau historique en France, l'Aide Sociale à l'Enfance s'est créée en 1943 afin de protéger mais aussi de surveiller les comportements des enfants en lien avec les difficultés parentales.

En 1986, l'Aide Sociale à l'Enfance est placée sous l'autorité du président du Conseil général (maintenant Conseil départemental ${ }^{1}$ ). Sa mission essentielle est de venir en aide aux enfants et à leur famille par des actions de prévention individuelle ou collective, de protection et de lutte contre la maltraitance. La loi du 5 mars 2007 réorganise la protection de l'enfance en développant des cellules de recueil des informations préoccupantes. Elle réaffirme les objectifs suivants: renforcer la prévention (détection de situations dites à risque), réorganiser des procédures de signalement et diversifier les modalités des prises en charge. Cependant, elle exige l'accord et la collaboration des parents et selon les associations comme Enfance et partage ${ }^{2}$, le système français soutient davantage les difficultés parentales et protégerait moins les enfants. La maltraitance intrafamiliale concerne $80 \%$ des situations (Benarous et al. 2014) et selon les chiffres de 2013, en France, 700 enfants meurent, tués sous les coups de leurs parents ${ }^{3}$. Lorsque le milieu familial n'est pas en mesure de garantir sa santé, sa sécurité ou sa moralité selon l'article 375 du Code Civil, ou si les conditions de son éducation ou de son développement physique, affectif, intellectuel et social sont gravement compromises ${ }^{4}$, le Juge des enfants peut prononcer une mesure d'assistance éducative, comme le placement d'un enfant. II confie alors l'enfant à la Direction Enfance et famille (structure départementale) qui va alors rechercher un lieu d'accueil (assistante familiale, lieu de vie ou institutionnel) susceptible de le soutenir dans son développement et de répondre à ses besoins affectifs, relationnels et physiques ${ }^{5}$.

Au regard de notre pratique clinique en France dans une maison d'enfants à caractère social ou foyer éducatif (MECS), accueillant et accompagnant des enfants et adolescents ayant connu des parcours de vie émaillés de séparation, ruptures et violences, nous avons choisi de

1. Le département est une division territoriale de la France. Leur création remonte au décret du 22 décembre 1789. Ils sont au nombre de 101, 96 d'entre eux sont en France métropolitaine.

2. 5 Amal Mogaïzel Parents criminels, l'omerta française, visionné le 5 août 2015, 20 :40$21: 30$, Programme tv PUBLIC SENAT - LCP.

6. Pour le lecteur, nous n'abordons pas les situations relevant de l'ordonnance 45 lorsque des mineurs ont commis une infraction qualifiée de crime ou de délit.

7. Placement d'un enfant sur décision judiciaire. Repéré à https ://www.servicepublic.fr/particuliers/vosdroits/F3140 
présenter une situation familiale originale. En effet, l'établissement accueille 3 fillettes issues de la même fratrie (âgées de 7 ans et demi pour la plus jeune, de 9 ans pour la cadette et de 10 ans pour l'aînée), placées sous décision judiciaire au regard de l'environnement familial négligent et violent. Cette situation est assez rare, car l'accueil des fratries en MECS n'est pas fréquent et en outre, dans un département du Sud de la France, les jeunes enfants sont habituellement placés dans des familles d'accueil et généralement, les adolescents, après de nombreuses ruptures de placement, sont pris en charge par les établissements sociaux de type MECS. II s'agit d'unités de vie pouvant accueillir jusqu'à 10 bénéficiaires avec des problématique sociales mais aussi présentant des fragilités psychiques ne leur permettant pas pour certains de suivre une scolarité, de mener un projet personnel ou encore de nouer des interactions de qualité avec autrui. Les études de Boden, Horwood et Fergusson (2007) montrent que les enfants maltraités obtiendraient moins de bons résultats scolaires et seraient orientés en éducation spécialisée (instituts thérapeutiques pour troubles du comportement ou encore classes d'intégration dans le système français). Précisons qu'en tant que psychologue dans cet établissement, nous n'assurons pas de suivi thérapeutique mais nous pouvons être amenés à rencontrer les enfants ponctuellement. Nous accompagnons également les équipes en repérant en quoi le positionnement éducatif peut impacter sa prise en charge et son comportement.

Après accord institutionnel pour mener cette recherche ${ }^{1}$, l'une d'entre nous a rencontré les fillettes. L'entretien clinique préalable visait à recueillir l'adhésion à la recherche mais surtout, dans un souci déontologique, de savoir si ces fillettes seraient en capacité de supporter la verbalisation des faits de maltraitances dont elles avaient été victimes. Conscientes des biais intrinsèques à notre écoute à visée de recherche, nous avons veillé à ce qu'elle ne se réduise pas à un simple recueil de faits ou de données cliniques et avons tenté de nous dégager d'une lecture événementielle, en nous centrant sur les capacités d'introspection et d'élaboration des conflits, du vécu traumatique, de façon partageable et cohérente. Nous avons créé un espace relationnel pour que l'enfant puisse s'exprimer avec confiance. Les effets de la subjectivité du chercheur (relation transféro-contre-transférentielle), sur le matériel clinique et

1. Nous avons demandé l'autorisation à la référente sociale de la situation (assistante sociale de secteur appartenant au conseil départemental) sous l'égide de sa directrice d'agence et les fillettes ont d'abord eu un premier entretien leur exposant avec des mots simples la recherche. Une semaine s'est écoulée avant qu'elles ne donnent leur accord écrit (formulaire de consentement que nous leur avons lu et expliqué au regard de leurs difficultés scolaires). La recherche a été ensuite expliquée au niveau institutionnel. Enfin la psychologue qui a rencontré les fillettes n'est pas celle qui assure le soutien psychologique. 
l'interprétation ont été analysés dans une optique de recherche certes, mais avant tout pour préserver l'enfant.

\section{Approche projective des maltraitances sexuelles infantiles et de la résilience}

La recherche de Condamin $(2006$; 2009) montre que le Rorschach participe à la compréhension des abus vécus par 100 jeunes patients. Ces enfants présenteraient un appareil à penser endommagé et se couperaient des apprentissages et du savoir. Le tableau clinique est très hétérogène car ils sont susceptibles de présenter des traits majeurs d'inhibition, narcissiques, des défenses primitives maniaques et comportementales (hyperinstabilité), une immaturité affective, une déficience intellectuelle, des traits psychotiques, des angoisses massives. En présentant deux cas paradigmatiques d'enfants ( 9 et 13 ans) ayant subi des agressions sexuelles, Mazoyer et Roques (2014) retrouvent au Rorschach une variété de traces traumatiques propres au fonctionnement psychique ainsi que des tendances générales référables à une fixation du trauma. Le processus associatif et les interprétations produites au Rorschach sont régulièrement altérés par des phénomènes projectifs comme l'expression de scènes d'agression, l'effraction des limites corporelles et psychiques, l'absence de représentations de relations humaines, des représentations de relations animales agressives, ou encore l'émergence de traumas plus anciens.

Dans une recherche exploratoire préalablement citée portant sur les enfants maltraités (résilients et non résilients), Lighezzolo, Marchal et Theis (2003) mettent au travail deux hypothèses : la première porte sur le tuteur de développement et la seconde sur les modalités défensives. Les auteurs interrogent le rôle joué à la fois par ce tuteur (ainsi que ses caractéristiques) et par les mécanismes de défense en jeu dans le processus de résilience. Pour ce faire, outre un entretien clinique semidirectif, ils soumettent les enfants à la passation d'un Rorschach et leur proposent de dessiner la personne qui a le plus compté pour eux. Cette méthodologie a permis de dégager chez les enfants résilients, l'investissement possible d'une personne rassurante sans idéalisation, et chez les enfants non résilients, la difficulté à s'identifier à un modèle structurant et à investir une relation duelle. Nous avons fait le choix de ne pas proposer de Rorschach au regard de l'inhibition retrouvée lors des premières rencontres chez les 3 fillettes. Nous avons opté pour des épreuves de dessin et un test thématique (CAT) qui proposent un support plus concret, plus figuratif, moins angoissant. 
Valeur projective du dessin et intérêt du dessin de la famille

Dans un article interrogeant la valeur du dessin dans l'appréhension des modalités de fonctionnement psychique de l'enfant traumatisé, Romano (2010) rappelle son intérêt dans la relation thérapeutique avec l'enfant abusé, blessée dans son altérité et sa subjectivité, en contribuant à sa restauration. Le dessin peut aussi offrir à l'enfant la possibilité de maitriser le trauma alors même qu'il est épreuve de passivité. II constitue un bon indicateur de son développement psychique et de sa dynamique affective.

Le dessin de la famille, comme test de personnalité d'inspiration psychanalytique créé par Corman $(1961 ; 1978)^{1}$, évalue le fonctionnement psychique de l'enfant et plus précisément les représentations de relations familiales. A travers son dessin, chacun y raconte les liens entre les personnes les proximités électives et les omissions révélatrices de sa position singulière dans le réseau familial (Brassac, Mietkiewicz, 2008). Corman $(1961,1978)$ attribue à ce test une grande importance puisqu'il permet à l'enfant d'exprimer sa conception de la vie familiale en dessinant une famille à sa convenance (selon ses désirs). Le clinicien observe comment l'enfant dessine la composition de sa famille et l'ordre de son dessin mais aussi la place accordée à chaque personnages. Les personnes oubliées ou ajoutées donnent un éclairage sur les sentiments des enfants. Ainsi, les personnages dévalorisés se caractérisent-ils par une taille diminuée. Ils peuvent être rabaissés, bâclés dans leur présentation, barrés, gommés ou oubliés. Par contre, la mise en valeur d'une personne dénote une relation significative. Souvent c'est un des parents qui est le premier représenté. La personne porteuse d'une charge affective importante est souvent dessinée en premier ou alors elle est plus grande et le dessin est soigné : l'enfant l'admire, l'envie ou la craint. Corman $(1961,1978)$ souligne que si l'enfant lui-même se dessine en premier, on peut parler d'un moindre investissement des images parentales souvent à la suite d'un conflit. Si un enfant (et pas l'enfant) est dessiné en premier, les principales aspirations narcissiques se cristallisent sur lui (place de choix dans la famille). Chez certains enfants, des réactions dépressives peuvent se traduire par l'absence de leur représentation dans le dessin.

1. La consigne appliquée consiste à demander à l'enfant: Dessine une famille, une famille que tu imagines ". Pour Corman, cette consigne donne libre cours à la fantaisie imaginative de l'enfant et permet aux tendances inconscientes de s'exprimer plus facilement.

Ensuite, l'enfant raconte l'histoire de la famille dessinée et termine en indiquant les personnes les plus gentilles, les plus heureuses, les moins gentilles et celle qu'il préfère parmi toutes. 
En outre, des parties oubliées comme les membres supérieurs dénotent une absence de contact et se sociabilité (Aubin, 1970). L'attention du clinicien se porte également sur la disposition spatiale et la distance des membres de la famille qui informent sur la nature et la qualité de leurs liens. Par exemple, est-ce que des sous-groupes dans la famille sont dessinés, comme par exemple, les petits séparés des grands ou encore les filles d'un côté, les garçons de l'autre?

\section{Le Children Apperception Test (CAT)}

Le CAT (Bellak, 1950) est le deuxième outil projectif que nous avons utilisé dans le cadre de notre recherche. II s'agit pour l'enfant, âgé de 6 à 8 ans et possiblement au-delà en fonction de sa maturité, de jouer symboliquement avec les images en faisant appel à divers degrés, au langage et à la pensée, la consigne étant de raconter une histoire à partir des 10 planches. Sur ces planches, sont dessinés, en noir et blanc plus ou moins estompés, des animaux familiers et sauvages dans des situations humaines quotidiennes (Bellak, 1950).

Le CAT permet d'appréhender la structure psychique, la dynamique des réactions de l'enfant devant les problématiques inhérentes à son développement et la façon dont il les résout. Cet outil projectif nous renseigne sur tout ce qui constitue le psychisme de l'enfant et sur le développement du Moi (Anzieu, Chabert, 1961, 1983) : les identifications, les conflits, l'angoisse, les mécanismes de défense, les interactions familiales mais aussi sur le niveau de maturité affective, notamment les modalités de la période de latence, en termes d'organisateurs de structuration œdipienne (différences des sexes et des générations) (Boekholt, 1993, 1998). Notre analyse des données cliniques obtenues au CAT s'appuie sur la grille élaborée par Boekholt $(1993 ; 1998)^{1}$ qui nous a permis de dégager des hypothèses métapsychologiques en fonction des problématiques latentes sollicitées par le contenu manifeste des planches (Anzieu, Chabert, 1961, 1983). Chez le jeune enfant, la projection sous sa forme verbale se manifeste par le recours à l'imaginaire et au fantasme (procédés IF), auxquels peuvent s'ajouter des procédés traduisant un recours à l'affect $(R A)$ et à la sphère motrice et corporelle $(M C)$. Ces procédés sont modulés par des procédés qui recourent à l'objectivation et au contrôle $(\mathrm{OC})$ et à la réalité externe $(\mathrm{RE})$.

1. M. Boekholt a construit une feuille destinée au dépouillement des trois épreuves thématiques verbales les plus utilisées dans la clinique infantile : le CAT, le Patte Noire et le TAT. Ces feuilles sont construites de façon à éviter tout risque de parti pris diagnostique afin de respecter la souplesse du psychisme de l'enfant.

II existe 7 rubriques: 1/Sphère motrice (MC). 2/ Relation au clinicien (RC). $3 /$ Recours à la réalité externe (RE). 4/ Evitement et inhibition (EI). 5/ Recours à l'affect (RA). 6/Recours à l'imaginaire et au fantasme (IF). 7/Objectivité et le contrôle (OC) 
Hypothèses, indicateurs des entretiens et des tests

\section{Hypothèses}

Afin de repérer les processus psychiques en jeu dans l'intégration d'un trauma d'origine familiale ayant conduit à un placement et la mobilisation de la résilience, nous posons les hypothèses suivantes :

1) La qualité de la capacité associative en entretien, au CAT et au dessin de la famille rend compte des tentatives d'intégration du trauma;

2) La résilience sororale permettrait à l'enfant d'investir le placement en prenant appui sur les autres membres de la fratrie.

Afin de dégager la mobilisation possible ou avortée de résilience sororale et de l'élaboration possible de l'événement traumatique, nous nous attarderons sur certains indicateurs repérables en entretien et aux épreuves projectives et dégagés à partir des travaux présentés en méthodologie.

1/ Chez l'enfant présentant une capacité à se dégager de l'impact traumatique et à intégrer l'événement douloureux :

- En entretien, les capacités de mentalisation sont repérables au travers de la qualité des associations des ressources défensives variées, notamment via l'expression d'affects modulés, associés à des représentations dans le discours

- Le dessin de la famille permet à l'enfant de revenir sur les configurations familiales pathologiques mais aussi de de souligner de nouvelles représentations de relation avec d'autres personnes hors famille mais considérées comme importantes pour lui. La représentation de soi n'est pas affectée. Le trauma n'a pas entravé l'établissement de relations objectales au profit d'un repli narcissique.

- Au CAT, les productions à partir des situations relationnelles générées par le matériel ainsi que les principales problématiques psychiques (identitaire, dépressive, œdipienne) n'apparaissent ni désorganisées ni désorganisantes (OC9: troubles de la syntaxe). L'enfant peut avoir recours à l'imaginaire et au fantasme (procédés IF3 : mise en scène, dialogue) tout en prenant en compte la réalité (OC1 : attachements aux détails).

1 bis/ Chez l'enfant sidéré par la violence de l'événement traumatique, du fait de l'enlisement dans l'événement traumatique :

- En entretien, la panoplie des ressources défensives s'avère restreinte. La projection et le déni viennent en lieu et place d'un refoulement opérant. L'altérité est menaçante et la relation à l'adulte semble teintée de méfiance, voire d'hostilité. 
- Au dessin de la famille, soit l'enfant peut basculer dans une idéalisation en montrant par exemple une famille heureuse et aimante (absence de conflits), soit il montre des signes de dépression ou d'une angoisse massive observable cliniquement par une instabilité psychomotrice ou des signes d'un refus ou d'une réticence l'empêchant de répondre de manière adaptée à la consigne.

- Au CAT, les productions peuvent rester très factuelles (procédés RE 1 : recours à l'évidence, RE2 : recours aux clichés), se contentant de décrire la planche sans forcément exprimer des actions. Le rapproché relationnel, surchargé dans la situation du CAT, peut donner lieu à des projections angoissantes, à une sexualisation des attitudes entre les personnages ou encore à des relations teintées d'emprise (IF7: fabulation, IF8 : expression crue, thématiques sexuelles et/ou agressives, IF9: confusion identitaire, télescopage des rôles).

2/ Chez l'enfant en capacité de prendre appui et de s'identifier à un autre de sa fratrie en vue de se développer :

- En entretien, soit il identifie un tuteur de développement en position de le protéger, soit il identifie un enfant plus jeune dont il faut s'occuper (capacité d'empathie). Nous serons attentifs au discours sur les autres enfants (placés, non placés) et sur le vécu singulier de cette situation de placement (être à trois dans la même institution).

- Au CAT, comme au dessin de la famille, l'analyse porte sur la représentation des relations, la régulation des conflits interpersonnels et l'élection d'un personnage auquel l'enfant s'identifie favorablement. Dans le dessin de la famille, nous retrouverons des échanges et des interactions (se parler, se regarder). L'enfant reconnaît une ou des personnes " amies ». Son récit développe des interactions familiales ou extrafamiliales (activités communes).

2 bis/ Lorsque l'enfant ne s'autorise pas à investir un autre dans une fonction de soutien, d'écoute, de protection :

- En entretien, peut assimiler tout adulte à un potentiel agresseur. II peut également manifester une grande indifférence dans ses relations, le repli étant un gage de sûreté narcissique au risque de désinvestir les relations. Le discours comme les productions peuvent être pauvres, ou marqués par la violence ou la crudité des représentations de relations.

- Au dessin de la famille, nous serons attentifs à la pauvreté des échanges et des interactions, à l'absence de personnage ami ou soutien dans le dessin de la famille. 
- Au CAT, l'enfant met peu les personnages en relation ou alors sous des modalités factuelles (procédés RE1 et RE2 au CAT), descriptives. La restriction prévaut $(E \mathrm{I}-1)$, l'anonymat des personnages est présent (El-2) et les conflits ne sont pas précisés (El-2). L'investissement narcissique prévaut dans un besoin de se protéger de la relation avec l'autre.

\section{Présentation des situations cliniques}

La famille des 3 fillettes (Elodie, Maurine et Florine, respectivement âgées de 10 ans, 9 ans et 7 ans et demi) est suivie depuis de nombreuses années par les services de l'aide sociale pour des carences éducatives importantes et une problématique de violences conjugale et sexuelle (suspicion d'attouchements d'un des frères). Les relations entre les différents intervenants et la famille sont limitées aux exigences liées à l'application de la mesure judiciaire exercée par un autre établissement. La famille est peu impliquée dans la mesure judiciaire. Les rencontres avec le père ou la mère sont minimes et les échanges ne permettent pas aux professionnels de créer une relation de confiance, d'engager un travail autour de la problématique familiale ni de soutenir la parentalité. Le nombre d'intervenants dans cette situation rend difficile une lecture plus approfondie du système familial.

M. et Mme ont eu ensemble huit enfants. Deux sont majeurs, hors domicile. Trois autres mineurs plus âgés que les fillettes rencontrées sont aussi placés mais séparément. Le placement des enfants a eu lieu dans un contexte de violence en lien avec un important conflit conjugal qui s'est soldé par la séparation des parents. Les fillettes ont assisté à des épisodes d'alcoolisation parentale et à des faits graves (incendie volontaire de la maison par le père alors qu'ils étaient dans la maison), pouvant mettre en danger leur vie. Le père et la mère ont noué de nouvelles relations amoureuses. Durant le placement, les fillettes ont repris les liens (visites médiatisées, liens téléphoniques) avec leur mère qui ne s'était pas du tout manifestée auprès d'elles pendant six mois.

Les enfants bénéficient d'un droit de visite et d'hébergement, en présence d'une travailleuse familiale en intervention sociale chez le père et chez la mère également depuis peu. Le juge des enfants a statué sur des droits de visite séparés au vu de la problématique familiale (suspicion d'attouchements/ violences sexuelles entre eux). Cependant, d'après les trois fillettes, les parents avec leurs conjoints respectifs du moment, se rencontrent tous les week-ends et elles vont et viennent chez l'un et l'autre indifféremment. Le cadre des droits de visite n'est donc pas respecté. 
Durant l'entretien, elles sont protectrices du père et de la mère, ne se livrant guère et communiquant peu d'éléments sur la violence intrafamiliale. Selon Lemay (2001, p. 142), « le désir de protéger l'image de l'agresseur afin de garder des traces identificatoires pour se construire conduit à vouloir rayer l'existence du traumatisme (...) ». Au niveau du foyer éducatif, les fillettes rencontrent des difficultés relationnelles, peuvent être l'objet de moqueries du fait d'une énurésie persistante (Florine, Elodie) et elles présentent des difficultés dans les apprentissages scolaires.

\section{Élodie, 10 ans}

En entretien. Dès le début de l'entretien, Elodie évoque des conflits avec un éducateur, ce qui lui permet d'exprimer un vécu complexe de placement. La fillette questionne les attitudes éducatives, en les jugeant " méchantes ", à l'exception de sa référente ${ }^{1}$ avec laquelle elle se sent en confiance.-On peut lire en filigrane un conflit de loyauté, la confiance ne pouvant être accordée qu'aux parents et à un substitut maternel. Elodie exprime des éléments de son vécu avec parcimonie et ne développe pas les relations intrafamiliales. Elle s'interdit de parler de sa séparation d'avec ses parents parler tout en énonçant sa difficulté à vivre sans eux. Elodie ne comprend pas les motifs du placement même si elle parle d'une maman débordée par le quotidien avec ses enfants. La fillette ne se vit pas comme soutien de ses sœurs et se dévalorise au regard des problèmes qu'elle rencontre (énurésie, difficultés scolaires). Elodie investit l'espace de la rencontre pour aborder le placement, son vécu, la douleur liée à l'éclatement de la cellule familiale et à l'absence de liens fraternels. Par contre, elle ne peut interroger ce qui a conduit à la séparation d'avec ses parents. Des affects de tristesse peuvent être reconnus même si la fillette réprime le plus souvent ses émotions.

Au CAT. Élodie propose des constructions narratives peu structurées et peu élaborées. Les personnages effectuent des tâches relevant du concret et du pragmatique ${ }^{2}$. L'espace imaginaire est inhibé, restreint, avec peu de résonance comme en témoigne un accrochage aux détails, et plus généralement à la réalité externe au détriment de la réalité interne (EI-1). Si les affects dépressifs ne sont pas verbalisés, nous pouvons quand même en déduire leur expression au travers de récits où l'éprouvé corporel

1. Le référent est l'éducateur attitré de l'enfant et serait l'équivalent d'un éducateur de suivi.

2. Planche 1 : ombre d'un coq ou d'une poule et 3 petits poussins devant un bol; planche 3 : un roi lion assis et une petite souris en bas de l'image; planche 4: maman kangourou sur un vélo et son petit dans la poche, petit kangourou lui aussi à vélo; planche 5 : deux petits ours dorment dans un petit lit, planche 6 : deux ours dorment dans une grotte et un petit se trouve devant. Dans les récits d'Elodie, la mère à la planche 1 attend, le lion à la planche 3 regarde dehors, à la planche 4 , la mère tient un panier, à la 5 et 6 , les ours dorment) 
et les postures traduisent la passivité («fatiguer », " dormir ») (Boekholt, 1998). Un vécu d'abandon se devine au regard du vide et de l'isolement relationnels que l'on peut observer à certaines planches, même si en fin de passation, on peut observer les prémisses d'une élaboration des conflits où les pulsions sont négociées au travers des représentations de relations. Cependant, les pulsions sont souvent censurées, aucune issue au conflit n'est proposée. L'excitation traumatique est traitée par un aménagement défensif rigide, ne permettant pas d'enclencher un quelconque travail d'élaboration. En outre, nous avons affaire à des interprétations relevant du registre archaïque où la violence et l'oralité prévalent et s'expriment par des angoisses de dévoration dans la relation parent-enfant comme par exemple à la planche $7^{1}$ : "Là c'est un tigre qui attaque...qui attaque un singe, un chimpanzé, dans la jungle et qui...qui va le manger comme il est carnivore ».

Au dessin de la famille. Elodie ne se saisit pas de la possibilité qui lui est offerte de laisser libre cours à sa créativité et à la fantaisie imaginative. Elle s'attelle à dessiner, telle une tâche scolaire et à reproduire à l'identique sa propre famille. Elodie s'investit dans la tâche demandée, elle s'attache à une certaine précision dans sa reproduction (place, nom et âge des personnes), même si le tracé est peu soigneux. Nous la soutenons par notre présence et notre regard dans la réalisation de son dessin dont elle paraît plutôt fière. Elodie représente les membres de sa famille sans aucune distinction vestimentaire. Tous de même taille et alignés, les personnages sont composés de bâtons pour l'ensemble du corps et d'une tête, niant la différence des générations. Seuls leurs âges et des cheveux longs pour les filles sont mentionnés. Elle dessine en premier son grand frère, Alexis qu'elle trouve d'ailleurs le plus gentil. Invitée à raconter l'histoire de cette famille, Elodie se vit comme la plus triste sans pouvoir en donner la raison. Tous sont décrits comme gentils mais à cette évocation, la petite fille se ferme et se réfugie dans le silence. La plus méchante est sa jeune sœur Florine (placée avec elle) qui a été dessinée en dernier. Elle est privée d'hamburger (on retrouve la prévalence de l'oralité repérée lors du CAT). Elle a hésité avant d'identifier sa sœur comme la moins gentille avec son frère Antonin susceptible de l'avoir agressée. Mais l'évocation du frère est vite suivie d'un silence et est balayée par le choix négatif final de sa jeune sœur. Ici, le recours au manifeste semble permettre à Elodie de mettre à distance le traumatisme activé par ses relations intrafamiliales.

\section{Maurine (9 ans)}

En entretien. Maurine peut se représenter la structure familiale même si elle évoque un demi-frère qui est en fait le compagnon de sa sœur aînée. Le divorce parental est très douloureux pour elle. Chaque fois que

1. Poursuite d'un singe par un tigre. 
Maurine évoque des moments tristes de son histoire, elle se trouve au bord des larmes sans possibilité de verbaliser davantage. Elle exprime une grande sensibilité et une capacité d'identification à la souffrance des autres, notamment à celle de ses sœurs : "les voir pleurer, ça me fait pleurer $»$.

Maurine peut aborder les évènements traumatiques et s'interroge sur le caractère intentionnel des actes. Des silences surviennent après l'évocation du trauma. Une désorganisation syntaxique montre toute la difficulté d'élaboration du traumatisme. En outre, elle cherche à donner un sens au trauma en tentant de comprendre la violence paternelle. A la fin de l'entretien, de manière défensive, elle annule la responsabilité paternelle (le père a pourtant été jugé et incarcéré) et le caractère intentionnel de l'acte. Les interactions familiales décrites restent marquées par des épisodes paternels et conjugaux d'alcoolisation. Maurine a aussi assisté à des violences conjugales dont le motif serait l'éducation des enfants et plus particulièrement le bruit qu'ils faisaient en jouant. Selon elle, la mère était battue à la place et à cause des enfants.

Maurine présente des capacités d'élaboration demandant à être étayées, à partir desquelles elle peut porter un regard sur sa situation, exprimer ses affects dysphoriques et un événement traumatogène, anxiogène. Les temps de visite avec ses parents sont évoqués de manière superficielle et désaffectivée, elle se montre capable de se projeter dans un futur rôle parental où elle pourra offrir à " son enfant tout ce qu'il veut ", ce qui nous semble relever d'une tentative de restauration narcissique.

Au CAT. Pour Maurine, cette épreuve projective favorise l'expression d'une problématique dépressive, d'abandon et de délaissement comme par exemple à la séquence de la planche 1: " y'a personne qui sert le plat ». Celle-ci s'accroche souvent au noir et au blanc, comme si cet accrochage sensoriel lui permettait de faire l'économie de l'expression d'affects dépressifs. L'insistance sur les qualités sensorielles du matériel renvoie à un vécu carentiel, un défaut de contenant maternel qui ne sauraient s'exprimer sans ce biais. La confrontation à la scène primitive est éludée par la répétition du verbe dormir et une description des qualités sensorielles de la planche (procédé RE4). L'insistance sur les éléments sensoriels de la planche (lit blanc, lit gris, noir et blanc) permet le déplacement sur l'environnement du besoin de repères consistants. La résonance dépressive en lien avec le vécu d'exclusion par le couple parental s'exprime par cette modalité. Ici, restriction et inhibition sont marquées par l'utilisation des procédés d'évitement où les silences et la nécessité de poser des questions indiquent une tendance à éviter toute conflictualisation (procédé El-2) par l'hyperinvestissement du perceptif. La fillette peut proposer de façon très ponctuelle des mises en relation entre 
un père et une mère. Elle se défend contre la curiosité pour la sexualité parentale («l'ourson est dérangé »).

La solitude peut être vécue avec terreur en raison de l'introduction de personnages anxiogènes et de l'apparition de fantasmes de dévoration ( le loup va la manger »). On note que le petit lapin du début du récit à la planche $9^{1}$ se féminise à la faveur d'une identification spéculaire, ce qui autorise la projection d'un vécu abandonnique. La dernière planche, évocatrice d'une relation parent-enfant dans un contexte d'analité, favorise une représentation paternelle de contrainte où le papa chien empêche le chiot de faire ses besoins. Le rapproché fantasmé père-fils donne lieu à des évocations de violence ( «il a très mal ...il veut s'enfuir ; le papa le tire »).

Au dessin de la famille. Maurine représente une famille imaginaire, intégrant des personnes qui l'entourent dans le foyer éducatif mais aussi des membres de sa famille réelle. Le nom donné à cette famille est à une lettre près son propre nom. La réalité aménage la projection. Au départ, elle se montre volontaire et appliquée dans la réalisation de son dessin et elle semble y prendre plaisir, puis elle accélère subitement le rythme de son dessin et " bâcle " sa production, en riant et en cachant sa production. Elle dessine au fur et à mesure des personnages de plus en plus difformes et ajoute un détail qui va produire chez elle un affect de honte et une excitation (des excréments). La façon dont les corps sont dessinés indique une certaine inhibition (traits petits, hachés, courts, et lignes rigides, droites avec peu d'amplitude) pouvant même indiquer une tendance au repli sur soi. Elle commente son dessin et précise parfois, au moment où elle les représente, l'identité des personnages. Mais celle-ci est instable, le frère devenant le papi, l'inconnu se transformant en mamie. L'instabilité de l'identité des membres de la famille, les nombreuses confusions et hésitations nous donnent à penser qu'il est difficile pour la fillette de se repérer dans un environnement familial suffisamment sécure. En termes d'identification, le bébé dessiné est à la fois le personnage le plus heureux mais aussi le moins gentil, ce qui atteste l'avidité et la rivalité de Maurine. Le personnage le plus bâclé est un garçon mais elle tait son identité et souhaite vite passer à autre chose. Le dessin de la famille est saturé en dimension anale (faire ses besoins, il va dans les WC), Maurine dessine des ronds qui représentent des excréments à tous les personnages, ce qui motive son rire durant toute l'exécution du dessin. Ce détail vient à la place d'une différence des sexes qui n'est pas marquée. La régression anale peut être investie comme défense face à la sexualité et pourrait aussi traduire une opposition et une agressivité peu maîtrisées. Les proportions de taille ne sont pas respectées, les adultes sont dessinés plus petits que

1. Un lapin dans son lit, la porte de sa chambre est entrouverte. 
les enfants, ce qui permet d'annuler la différence des générations. L'absence de mains renforce le manque de relations entre les personnages. Elle ajoute des personnages extérieurs à la famille afin d'exprimer le sentiment d'exclusion et de punition (" c'est un inconnu...qui pleure parce que son papa et sa maman ils l'ont viré »). L'évocation de cette thématique d'exclusion qui fait écho au fort sentiment d'abandon éprouvé est ensuite déniée " c'est pas ma famille ». Le dessin de la famille permet de repérer des mouvements d'idéalisation parentale ( " la mère la plus belle, ils donnent de l'argent et tout »), lesquels contre-investissent des éprouvés plus angoissants, saturés en oralité : " c'est des ogres...les grands ».

\section{Florine (7 ans)}

En entretien. Au quotidien, Florine est une enfant spontanée, à l'aise dans sa relation à l'adulte. Dans la relation transféro/contretransférentielle, elle recherche un cadre et semble tester les limites. Lors du premier entretien, la plus jeune des fillettes placées se montre réticente à l'échange et met fin à l'entretien en promettant de revenir. La situation de rencontre semblait trop intrusive et elle tente d'y échapper en adoptant une attitude séductrice. Rappelons que les enfants ayant connu des actes de maltraitance ou de carences vivent de façon intrusive et séductrice l'invitation à parler émanant de l'adulte. Au regard d'une réalité familiale traumatique, on peut se demander si Florine a perçu le clinicien comme voyeuriste, la passation d'épreuves projectives activant des configurations fantasmatiques de ce type. Toutefois, la question d'une éventuelle censure familiale peut aussi être posée, d'un interdit « de parler » par identification à la mère, elle-même fermée et peu dans la collaboration avec l'équipe éducative. Florine n'aborde pas vraiment la problématique familiale, ne s'exprime pas sur le climat de violences auquel elle peut être confrontée et idéalise les figures parentales et les liens familiaux.

Si Maurine a abordé et questionné la violence paternelle et tenté de mettre du sens sur un acte qui aurait pu avoir des conséquences terribles (mort des enfants), Florine, lors d'un second entretien, évoque des faits de séduction de son frère sur sa sœur Elodie. Cette agression est peu élaborable et les places de victime/agresseur non repérables. L'excitation se manifeste par un fort trouble de la syntaxe où transparaît une confusion de personnages : « il avait fait un bisou sur la bouche à Elodie (placée et rencontrée) et là elle a continué, et après c'est moi et Ophélie (leur plus grande sœur) qui a...qui a...qui a pris ». L'interdit ne semble pas non plus intégré. Selon elle, le placement a été prononcé en raison de cet épisode, ce qui empêche des mouvements pulsionnels agressifs dirigés vers les parents. Elle rend responsable sa sœur Elodie du placement et de la rupture avec leur grande sœur qui ne souhaiterait plus les voir. 
A plusieurs reprises, la dernière des filles souhaite avoir la photocopie de nos notes ${ }^{1}$ ( " personne veut m'écrire ») et critique l'action des professionnels (« vous, vous faites rien »). Elle peut exprimer être l'objet de moqueries des autres enfants en raison de son embonpoint et de son énurésie. Elle insiste sur la douleur d'être séparée de ses parents et l'envie de retourner auprès d'eux. La colère, l'agressivité envers les autres sont activées lors de situations où elle se sent blessée. Le recours au comportement tempère l'expression et la reconnaissance d'affects dysphoriques.

Au CAT. La difficulté préalable, ici pour la passation, a été de susciter l'intérêt de Florine et surtout de la maintenir durant l'épreuve, au regard de l'instabilité psychomotrice et des difficultés de concentration dont elle peut faire preuve (procédé MC2). Cependant, Florine s'est très vite approprié le matériel, comme à son habitude dans les entretiens précédents (stylo, feuille, dictaphone). Elle a beaucoup manipulé les planches tout au long de l'épreuve. L'activité projective, soutenue par un travail de mise en scène et de dialogues, signe l'existence d'une scène psychique où les personnages s'animent. Le rapproché est l'occasion de déployer un lien libidinal entre une mère et son enfant (planche 10). L'expression conflictuelle n'est pas traitée à chaque planche, l'évitement et l'inhibition empêchant parfois Florine d'aborder les conflits autour d'un récit cohérent et structuré. Le plus souvent, la conflictualisation est refusée, puis le mouvement d'inhibition est levé partiellement : « on voit pas », « rien, il se passe rien ». La montée de l'angoisse en lien avec le contenu latent des planches est lisible au travers de l'instabilité psychomotrice comme aménagement défensif répétitif.

Cependant, une précarité identitaire peut être repérée : notamment à la planche 1 , les poussins sont d'abord perçus comme des pigeons, l'identité sexuée est soumise à hésitation car le coq devient une poule. II est difficile de suivre l'identification des personnages par Florine. La perception d'une planche avec plusieurs personnages à la $8^{2}$ donne lieu à une dilution du sentiment d'identité comme si Florine n'arrivait pas à discriminer la différence dans un groupe élargi, ce qui questionne la place de la fillette dans sa fratrie élargie.

Le verbe dormir permet de taire les manifestations pulsionnelles éveillées par les représentations parentales. La problématique anale est

1. Pour cette fillette, avoir la photocopie des notes pour les mettre dans son dossier s'inscrit dans le besoin de garder une trace comme si elle craignait l'effacement et l'oubli : " pour que j'ai des trucs de maman ». Ce qui questionne la permanence de l'objet et la symbolisation, soit penser en l'absence de l'objet.

2. Le contenu manifeste (ce qui se voit) : un singe au premier plan parle à un petit singe. Deux grands signes sont assis et boivent dans des tasses. Au niveau des sollicitations latentes, l'enfant est invité à se situer dans le groupe familial et à repérer les relations entre les différents membres. 
souvent convoquée en lieu et place de la sollicitation latente autour de la curiosité en lien avec la scène primitive. L'intérêt pour la sexualité parentale est déplacée sur les contenus corporels (découverte autoérotique : faire pipi). Du fait de son plus jeune âge, l'aménagement défensif de Florine repose essentiellement sur un mode de décharge par les voies motrices et corporelles (les verbes d'action au CAT sont nombreux: sauter, se cacher, aller), ses capacités actuelles de mentalisation restant insuffisantes ne lui permettant pas d'enclencher un travail de transformation de l'excitation pulsionnelle propice à l'accès au processus de résilience.

Au dessin de la famille. La consigne est mal acceptée par la fillette et nécessite d'être aménagée pour qu'elle l'accepte : " je veux pas dessiner une famille parce que c'est long $»$. Elle accepte de dessiner finalement une famille de chats. Le dessin est assez vite exécuté et Florine veut quitter rapidement la situation de bilan psychologique. Les chats sont dessinés de façon sommaire, avec des corps disproportionnés (petites têtes et longs corps), et une absence de méticulosité, les traits étant irréguliers et grossiers, le dessin est à la limite du débordement. Invitée à raconter I'histoire de cette famille, Florine établit des interactions verbales (elle exprime les liens, les relations par des flèches entre les membres de la famille, donnant vie aux personnages) mais l'hyperinstabilité des identifications se traduit par le changement brusque de personnage comme si Florine ne pouvait s'identifier de façon préférentielle à un personnage : « La maman elle dit bonjour à son enfant « bonjour mon petit chaton » et le chaton il va voir son papa et il lui dit "ça va? » ...le papa il va voir son petit et il lui dit bonjour et le p'tit il va voir sa grande sœur et lui dit "salut meuf, ça va? " et la grande sœur elle va voir son petit " oui ça va ». Il est à noter que le personnage de la grande sœur est investi dans une dimension maternelle. Dans un premier temps, Florine semble s'identifier au " chat bébé » en demande d'attention et de soins maternels. Celui qui est le plus heureux est le bébé chat car il mange. La régression et l'oralité lui permettent de retrouver une position privilégiée et un rapprochement exclusif avec ses figures parentales, évitant ainsi tout conflit anxiogène.

A la fin de la passation, Florine insiste pour avoir la photocopie du dessin et exprime colère et ressentiment à l'endroit des adultes. Ce dialogue est aussi l'occasion d'attaquer l'asymétrie des places entre une enfant et une adulte en situation professionnelle. 


\section{DISCUSSION DES RÉSULTATS}

\section{Élaboration et intégration du traumatisme}

Les fillettes ont investi l'espace de la rencontre pour dire leur douleur d'être séparée de leur famille. Seule Maurine et à un niveau moindre Florine, sont revenues sur les interactions familiales et sur une réalité traumatique notamment en évoquant les actes dont ont été coupables le père et le frère.

Le travail de mentalisation (soit l'élaboration mentale et l'expression affective) est suspendu au profit d'une charge émotionnelle difficilement canalisable pour Maurine (pleurs) et pour Florine (agressivité, attaque du cadre). Au regard de l'exposition à divers types de trauma depuis leur petite enfance, il semblerait qu'elles n'aient pas développé de capacité de mentalisation et de symbolisation (ce qui expliquerait l'hyperinvestissement des éléments factuels). La séparation imposée judiciairement a révélé les carences familiales, l'absence de protection parentale, mais a aussi provoqué chez les fillettes des interrogations quant à leur appartenance à la famille, ce qui pourrait expliquer les résistances au dessin de la famille. Notons toutefois à cette épreuve que les échanges et les interactions entre les personnages sont très limités et que la fantasmatique archaïque est prévalente (oralité, analité). II est à préciser que les mouvements d'idéalisation de la famille et des parents peuvent aussi traduire une culpabilité, notamment chez Elodie qui se rend responsable du placement (les agissements de son frère ont accéléré le placement).

\section{À propos de la résilience sororale}

Dans la littérature scientifique, le lien fraternel peut venir suppléer les défaillances parentales par la construction d'un nouveau lien d'attachement entre les membres de la fratrie où s'organise une régulation des conflits fraternels, des rôles et places de chacun qui unissent plus qu'ils ne divisent. Cette forme de résilience permet à la fratrie de former une carapace groupale afin de résister à la violence externe qui lui est faite et de surmonter leur souffrance.

Au regard des données cliniques recueillies en entretien, au CAT et au dessin de la famille des trois sœurs rencontrées, il semblerait qu'à la défaillance parentale s'ajoute une fluctuation des places de chacun. Les deux aînés encore à la maison (Alexis, 15 ans et Ophélie, 17 ans) semblent avoir été ponctuellement investis par les autres comme substituts des figures parentales mais dans des fonctions partielles (" garder", " s'amuser avec »). Cependant, dans les faits, aucune des sœurs ne revoit Ophélie qui a décidé de couper les liens avec elles suite à l'épisode d'attouchement sur Elodie. Elle était ce jour en charge de surveiller ses jeunes sœurs mais s'était endormie. Toutefois, dans le contexte de 
placement et de séparation familiale, il semblerait que le manque de repères identificatoires des sujets, la confusion des places de chacun ne favorisent pas un quelconque étayage mutuel entre eux, ni l'édification d'une suppléance pour pallier les défaillances parentales passées et présentes. L'évitement à aborder les relations familiales en entretien et l'isolement des personnages dans les épreuves projectives montrent le repli narcissique et la difficulté à investir l'autre. Les trois sœurs peuvent manifester aussi une certaine agressivité dans leur proximité : elles partagent peu de moments de complicité (insultes, bagarres). Chacune, à un moment donné, a pu exprimer une volonté d'autonomie, un désir d'être seule et différenciée du groupe fraternel. Plus qu'une réelle empathie, une identification narcissique, soit en miroir, semble en jeu (être triste lorsque l'autre pleure). Ne reconnaissant pas le rôle du tiers, l'identification narcissique ne s'inscrit pas dans un système triangulaire mais duel et cherche à colmater les éprouvés de perte. Ainsi, dans cette situation, les potentialités du groupe fraternel placé ne sont-elles pas propices à la construction d'une réelle entité dont la force et la résistance leur permettrait de surmonter l'adversité. L'épreuve de solidarité, à laquelle leur situation les confronte, n'engendre pas actuellement (au vu de leur jeune âge) un processus de résilience sororale. Cependant, il n'est pas exclu qu'au fur et à mesure de l'éloignement d'avec les parents et d'autres membres toxiques de la fratrie et de l'avancement dans l'âge, les liens entre sœurs ne se développent, ne deviennent de meilleure qualité, et favorisent une forme de résilience sororale. II semblerait opportun de suivre ces jeunes filles dans le cadre d'une étude longitudinale conciliant recherche et prise en charge psychothérapeutique en parallèle. Ce soutien thérapeutique soutiendrait la recherche de sens aux expériences traumatiques et l'identification et la reconnaissance des émotions chez les fillettes. Au regard des problèmes relationnels rencontrés sur le groupe de vie, cet espace de parole viserait possiblement avec le recours à diverses médiations thérapeutiques (jeu...) à tenter de comprendre et de résoudre certains conflits interpersonnels.

\section{CONCLUSION}

Dans cette recherche, nous souhaitions comprendre et analyser par quels processus psychiques un enfant surmonte une situation d'adversité. Comment et par quels aménagements parvient-il à se dégager de l'impact traumatique? En quoi la résilience sororale peut-elle constituer une voie de traitement du trauma? Nous pouvons à partir de cette recherche, repérer des manifestations du trauma chez les fillettes rencontrées. Chez elles, le placement est vécu comme une punition et les négligences parentales et familiales en amont de celui-ci ne sont pas ou peu exprimées. En outre, l'idéalisation parentale assez agissante rend difficile la mobilisation de la résilience sororale. D'autre part, la dynamique familiale maltraitante et 
négligente a, semble-t-il, impacté sur les interactions et complexifié la représentation de l'autre chez ces fillettes. Actuellement, l'investissement narcissique (de soi ou d'un équivalent comme un parent) prévaut au détriment de l'investissement objectal (de l'autre). Le placement dans un même lieu n'a pas renforcé la résilience sororale, même si Elodie peut adopter une position de consolatrice envers ses sœurs. Cependant, elle est considérée et se vit comme la responsable du placement car des actes sexuels ont été perpétrées sur elle et ont accéléré la décision judiciaire. Selon nous, les attouchements sexuels du frère font écran au contexte de négligence et de violence familiale. La difficulté à questionner le fonctionnement familial maltraitant et négligent pourrait mettre à terme le placement en échec, car les fillettes ne verraient pas de sens à la prise en charge et demeureraient prisonnières des difficultés familiales. Par ailleurs, il semble nécessaire d'instaurer une relation qui ne soit pas fondée sur les retrouvailles autour d'un mauvais objet, soit l'institution, faisant l'économie d'un vrai travail psychique sur un vécu traumatique.

Notre action en tant que professionnels de l'aide sociale à l'enfance oscille entre deux risques : celui de penser que le parent sera transformé magiquement par la séparation d'avec son enfant et d'autre part, de penser toute action en dehors de lui, de se représenter l'institution comme supplétive de la famille ${ }^{1}$. Les rencontres cliniques avec les trois sœurs soulignent le déficit de confiance en l'adulte éducatif et leur ont permis d'exprimer la douleur d'être séparées de leurs parents. L'espace de la recherche a permis à ces fillettes d'exprimer la douleur de ne voir leurs parents que sur des temps courts. Cette situation exige de nous questionner quant à la pertinence du placement familial et surtout à son idéologie française ${ }^{2}$ et à réfléchir aux échecs de placement (par exemple des passages à l'acte se soldant par une exclusion institutionnelle) par manque d'adhésion de la famille à la mesure.

D'autre part, cette recherche souligne la contribution des épreuves projectives (ici CAT et dessin de la famille) afin de souligner l'impact psychique de la maltraitance mais aussi de repérer les capacités de mentalisation en vue du traitement des problématiques et des conflits constitutifs autour de l'identité, de la filiation, de la capacité dépressive. Certains enfants victimes de maltraitance évitent toute situation évocatrice de maltraitance ou d'abus. L'entretien clinique peut réactiver des

1. Scelles (2004) souligne la tentation et les attitudes chez le professionnel de " tout faire » (réparer), de " trop faire » (ne pas laisser le temps de faire) et de " ne pas assez faire » (laisser le parent dans une profonde solitude).

2. Les travaux de Berger (2003) montrent combien les professionnels peuvent davantage s'identifier à la souffrance de parents qu'à celle de l'enfant et à la terreur ressentie en leur présence. Les projets institutionnels (retour de l'enfant au domicile parental, échecs itératifs de placement) peuvent coller à une demande de réparation narcissique des parents sans une réelle prise en considération des besoins de l'enfant. 
souffrances en réactualisant des scènes traumatiques encore vives, or dans le cadre de la passation d'épreuves projectives comme le CAT ou encore le dessin de la famille, l'enfant n'est pas contraint d'aborder directement un vécu douloureux. Ces médiations facilitent la relation avec le clinicien, relation qui est souvent entachée de perte de confiance envers les adultes. Elles donnent accès au monde interne et permettent d'évaluer les retentissements du trauma sur la réalité psychique et notamment la qualité des représentations adressées à un autre. Le processus associatif est donc soutenu par une relation transférentielle. Enfin, les médiations projectives déplacent un regard professionnel focalisé sur des attitudes et des comportements pour s'intéresser à la réorganisation de la personnalité après un traumatisme et aux ressources personnelles mobilisables. En d'autres termes, elles favorisent une mobilisation de l'aire transitionnelle (Winnicott, 1969) (trouver/créer, articulation entre perceptif et projectif), de l'expression des relations et des interactions, le traitement de l'angoisse et des conflits, la qualité des identifications. Le travail du clinicien avec l'enfant placé consisterait à lui offrir les moyens de se structurer psychiquement dans la situation de séparation qui est la sienne. Et ce, afin de pouvoir développer un système défensif solide et d'engager un processus de résilience, quitte à investir des tuteurs (hors famille) rencontrés au gré des rencontres.

\section{RÉFÉRENCES}

Anzieu, D., \& Chabert, C. (1961). Les méthodes projectives. Paris: PUF, 2004, coll. Quadrige.

American Academy of Child and Adolescent Psychiatry Official Action. (1998). Practice parameters for the assessment and treatment of children and adolescents with posttraumatic stress disorder. Journal of the American Academy of Child and Adolescent Psychiatry, 37(suppl10), 4-26.

Anaut, M. (2002). Trauma, vulnérabilité et résilience en protection de l'enfance. Connexions, 1(77), 101-118.

Arnaud, M. (2003). Forme de résilience dans le groupe fraternel. Le Divan familial, 1(10), 123-131.

Aubin, H. (1970). Le dessin de l'enfant inadapté; significations et structures. Toulouse : Privat.

Bailly, L. (2001). Syndromes psycho-traumatiques chez l'enfant. In M. De Clercq \& F. Lebigot (Éds), Les traumatismes psychiques (p. 137-147). Paris : Masson.

Bellak, L. (1950). An Introductory note on the Children' Apperception Test (CAT). Journal of Projective Techniques, 14, 215-234.

Benarous, X., Consoli, A., Raffin, M., \& Cohen, D. (2014). Abus, maltraitance et négligence : (1) épidémiologie et retentissements psychiques, somatiques et sociaux. Neuropsychiatrie de l'Enfance et de l'Adolescence, 62, 299-312.

Berger, M. (1992). Les séparations à but thérapeutique. Toulouse : Privat.

Berger, M. (2003). L'Enfant et la souffrance de la séparation. Paris : Dunod.

Boden, J. M. Horwood, L. J., \& Fergusson, D. M. (2007). Exposure to childhood sexual and physical abuse and subsequent educational achievement outcomes. Child Abuse \& Neglect, 31, 1101-1114.

Boekholt, M. (1993). Épreuves thématiques en clinique infantile. Approche psychanalytique. Paris : Dunod

Brassac, Ch., \& Mietkiewicz, M-C.(2008). La production conjointe d'un dessin de la famille : une histoire interactionnelle. Bulletin de psychologie, 3(495), 245-255. 
Condamin, Ch. (2006). Corps démembré, corps supplicié, corps massacré. Le Rorschach chez les enfants et adolescents victimes d'agression. Champ psychosomatique, $1(41), 129-142$.

Condamin, Ch. (2009). Trauma, résilience et mémoire du corps: A propos d'un cas d'un enfant sexuellement maltraité, Approche projective et théorico-clinique. Bulletin de psychologie, 503, 457-466.

Cyrulnik, B. (1999). Un merveilleux malheur. Paris : Odile Jacob.

Corman, L. (1961). Le test du dessin de famille. Paris : PUF.

Ferenczi, S. (1919). Psychanalyse des névroses de guerre. O.C. III, Psychanalyse 3. Paris : Payot, 1982.

Ferenczi, S. (1932). Confusion de langue entre les adultes et l'enfant. Paris: Payot, coll. Petite Bibliothèque Payot, 2004.

Ferenczi, S. (1934). Le traumatisme. Paris : Payot, coll. Petite Bibliothèque Payot, 2006.

Fonagy, P. Steele, M., Steele, H, Moran, G. S., \& Higgitt, A. C. (1991). The capacity for understanding mental states: the reflective self in parent and child and its significance for security of attachment. Infant Mental Health Journal, 12(3), 201-218.

Fonagy, P., Gergely, G., Jurist, E. L., \& Target, M. (2004). The development of an understanding of self and agency. In. P. Fonagy, G. Gergely, E. L., Jurist, M., \& Target (Éds), Affect regulation, mentalization, and the development of the self (p. 203-251). New York, NY : Other Press.

Fonagy, P., \& Target, M. (2006). The mentalisation-based approach to self pathology. Journal of Personality Disorders, 20, 544-576.

Freud, S. (1920). Au-delà du principe de plaisir. Paris : PUF, coll. Quadrige, 2013.

Freud S. (1926). Inhibition, symptôme et angoisse. Paris : PUF, coll. Quadrige, 2011.

Janin, C. (1996). Figures et destins du traumatisme. Paris : PUF.

Laplanche, J., \& Pontalis, J. B. (1967). Vocabulaire de la psychanalyse. Paris : PUF.

Lemay, M. (2001). La résilience devant la violence. Revue québécoise de psychologie, 22(1), 135-148.

Lighezzolo, J., Marchal, S., \& Theis, A. (2003). La résilience chez l'enfant maltraité : « tuteur de développement " et mécanismes défensifs (approche projective comparée). Neuropsychiatrie de l'enfance et de l'adolescence, 51(2), 87-97.

Manciaux, M. (1993). L'enfant maltraité. Paris : Éditions Fleurus.

Mazoyer, A-V, \& Roques, M. (2014). Mobilisation des processus psychiques chez des enfants victimes d'agression sexuelle. Contributions du Rorschach à la clinique du trauma. Bulletin de psychologie, 4(532), 331-348.

Mogaïzel, A. (2014). Parents criminels : l'omerta française. Documentaire diffusé le 5/8/2015 à $20 \mathrm{~h} 30$ sur la chaîne française LCP, durée 52 minutes.

Romano, H. (2010). Le dessin-leurre. La psychiatrie de l'enfant, 53, 71-89.

Scelles, R. (2004). Penser la fragilité et les compétences des parents pour ouvrir sur des pratiques qui soient « bientraitantes ». Pratiques psychologiques, 10(1), 1-5.

Scheeringa, M. S., \& Zeanah, C. H. (2001). A relational perspective on PTSD in early childhood. Journal of Traumatic Stress, 14(4), 799-815.

Taïeb, O. Baubet, T. Pradère, J. Lévy, K. Revah-Lévy, A. Serre, G., \& Moro M.-R. (2004). Traumatismes psychiques chez l'enfant et l'adolescent. EMC-Psychiatrie, 1, 23-32.

Terr, LC. (1991). Childhood traumas: an outline andoverview. The American Journal of Psychiatry, 148, 10-20.

Winnicott, D. W. (1969). Objets transitionnels et phénomènes transitionnels. In D. W. Winniicott (Éds), De la pédiatrie à la psychanalyse (p. 109-125). Paris : Payot.

\section{RÉSUMÉ}

Après avoir rappelé les principales théories du traumatisme, nous analysons les effets psychiques des maltraitances et la mobilisation de la résilience de type sororal auprès de 3 jeunes sœurs placées dans un foyer éducatif. Grâce à une méthodologie projective (entretiens cliniques de recherche, Children apperception test et un dessin de la famille), les résultats montrent que le trauma peine à être intégré par la fratrie et que la résilience sororale 
ne s'est pas développée. Cet article affirme la nécessité de soutenir une réflexion autour de placement de membres d'une même fratrie.

\section{MOTS CLÉS}

enfants maltraités, trauma psychologique, résilience sororale, élaboration, épreuves projectives, placement

\section{ABSTRACT}

After reviewing main theories about psychological trauma, we study psychological effects of maltreatment and sibship resilience in three young sisters met in a French special education institution, using a projective methodology (clinical research interviews, Children's Apperception Test and family drawing). Results show that the traumatism is still active and that sibship resilience did not occur. This research underlines the importance of persistence in the reflection about sibs in state foster care.

\section{KEY WORDS}

child abuse, psychological trauma, sororal resilience, elaboration, projective tests, foster home placement 